\title{
Tracing Cosmic Dawn
}

\author{
Anastasia Fialkov \\ Harvard-Smithsonian Center for Astrophysics, Institute for Theory and Computation, \\ 60 Garden Street, Cambridge, MA 02138, USA \\ email: anastasia.fialkov@cfa.harvard.edu
}

\begin{abstract}
Observational effort is on the way to probe the 21-cm of neutral hydrogen from the epochs of Reionization and Cosmic Dawn. Our current poor knowledge of high redshift astrophysics results in a large uncertainty in the theoretically predicted 21-cm signal. A recent parameter study that is highlighted here explores the variety of $21-\mathrm{cm}$ signals resulting from viable astrophysical scenarios. Model-independent relations between the shape of the signal and the underlying astrophysics are discussed. Finally, I briefly note on possible alternative probes of the high redshift Universe, specifically Fast Radio Bursts.
\end{abstract}

Keywords. Cosmic Dawn, Reionization, 21-cm signal

\section{Introduction}

The time in the cosmic history between the decoupling of the Cosmic Microwave Background $(\mathrm{CMB})$ radiation at redshift of $z \sim 1100$ and the appearance of the rich population of observed galaxies in the local Universe (out to $z \sim 11$, Oesch et al. (2016)) remains veiled in mystery. The first population of sources existing at the dawn of the Universe are too faint to be directly observed by existing telescopes. This is unfortunate because Cosmic Dawn is marked by unique events such as the formation of the very first stars, galaxies and black holes. As a result of our ignorance on the process of galaxy formation at early times, theoretical models are poorly informed and predictions for the astrophysical signals vary within few orders of magnitude. In particular, this applies to the 21-cm signal which is produced by neutral hydrogen atoms in the intergalactic medium (IGM). Intensity of the produced line depends on the underlying density of the gas as well as on the radiative backgrounds emitted by first stars and black holes, which define the thermal and ionization state of the gas.

The expected shapes of both the global 21-cm signal and the power spectrum of its fluctuations carry information about events in cosmic history from the entire period between recombination and reionization. These signals can be used as cosmic clocks indicating, at every moment of cosmic history, which exactly astrophysical processes were important. Specifically, the signal from the epoch prior to star formation is fully determined by atomic physics and cosmology: when the gas is dense enough, collisions couple the spin temperature of the 21-cm transition to the temperature of the gas, and the signal is seen in absorption. When the Universe expands enough and the gas rarefies, the collisions become inefficient and the coupling to the CMB dominates. This process drives the spin temperature of the transition to the temperature of the CMB and the contrast between the 21-cm spin temperature and the CMB decreases. As soon as the first stars form, they produce Ly- $\alpha$ background which couples the spin temperature back to the temperature of the gas via the Wouthausen-Field effect (Wouthuysen (1952), Field (1958)). In the absence of heating sources, the gas is much colder than the CMB and the signal is sen in absorption. Once X-ray sources form (e.g., accreting black holes and black hole binaries) IGM is heated up and above the temperature of the CMB. The onset of the heating 
era marks the deepest point in the absorption trough. If the heating is strong enough, the signal will be seen in emission at the later stages. Finally, the process of ionization becomes dominant and drives the 21-cm signal to zero depleting the HI reservoirs.

\section{Astrophysics of the Early Universe}

In Cohen et al. (2017) and Cohen et al. (2017) we varied five key astrophysical parameters and explored the range of the resulting 21-cm signals. Because of the lack of observations, astrophysical parameters are poorly constrained resulting in a large variety of 21-cm signals. Specifically, we explored the following parameters: (1) Star formation efficiency, $f_{*}$, defined as the ratio between the stellar mass to halo mass. Simulations show that $f_{*}$ in first star forming halos can be anywhere below $50 \%$ (Wise et al. (2014), O'Shea et al. (2015) and Xu et al. (2016)). (2) Minimal circular velocity of the star forming halos (directly related to the minimal mass of halos that can radiatively cool gas and form stars) can be anywhere between $V_{c}=4.2 \mathrm{~km} / \mathrm{s}$ which corresponds to the molecular hydrogen cooling channel (Tegmark et al. (1997)) and $V_{c} \sim 75 \mathrm{~km} / \mathrm{s}$ which corresponds to $M_{h} \sim 10^{9} \mathrm{M}_{\odot}$, e.g., if star formation is suppressed due to supernovae or photoheating feedbacks. (3) The total optical depth of the CMB, $\tau$. According to the measurement by Planck $\tau \sim 0.055 \pm 0.009$ (Planck Collaboration (2016)); however, higher values are favored if ionization history is gradual Miranda et al. (2017). (4) X-ray heating is another poorly constrained process. Several parameters related to the population of X-ray sources are important, namely the spectral energy distribution (SED) and (5) luminosity, $L_{X}$. Heating can be due to sources with hard SED (X-ray binaries or miniquasars), soft SED (hot gas in galaxies) or a mixture of the above (Fialkov et al. (2014) and Pacucci et al. (2014)). Parameter $f_{X}$ is the measure of the bolometric luminosity per star formation rate, $L_{X} / S F R$, in units of the observed luminosity at low redshifts. The upper and lower limits on $f_{X}$ characteristic to the pre-EoR population depend on the redshift at which reionization is completed (Fialkov et al. (2017), Pober et al. (2015)). Both population synthesis simulations (Fragos et al. (2013)) and observations (Brorby et al. (2016)) suggest that in the case of X-ray binaries, $L_{X}$ is larger in metal-poor high-redshift galaxies compared to their metal-rich low-redshift counterparts. The upper limit on $f_{X}$ is derived using the unresolved X-ray background observed by Chandra (Fialkov et al. (2017)) and is $\sim \mathcal{O}(100)$ or higher depending on the redshift of reionization.

\section{Experimental Constraints}

Existing radio telescopes have started to rule out viable astrophysical scenarios. In particular, experiments targeting the EoR, including the Experiment to Detect the Global EoR Signature (EDGES, 90-190 MHz, Monsalve et al. (2017)) and the Shaped Antenna measurement of the background RAdio Spectrum (SARAS, 110-200 MHz, Singh et al. (2017a) and Singh et al. (2017b)), rule out scenarios with inefficient heating, strong Ly- $\alpha$ and sudden reionization. On the other hand, Cosmic Dawn experiments such as the LargeAperture Experiment to Detect the Dark Ages (LEDA, Price et al. (2017)) have potential in near future to constrain models with early star formation and efficient heating.

\section{Parameter Study: Lessons Learned}

The large allowed parameter space outlined above leads to a large scatter in the expected 21-cm signals, which we discussed in details in Cohen et al. (2017) and Cohen et al. (2017). The parameter study is based on $\sim 200$ different astrophysical scenarios 
which bracket the entire parameter space. The main lessons of this parameter study include the following:

- The uncertainty in the power spectrum is $\sim 4$ orders of magnitude at scales of 0.1 $\mathrm{Mpc}^{-1}$; while the depth and position of the absorption trough can be anywhere within $\nu=40-120 \mathrm{MHz}$ and $\delta T_{b}=25-240 \mathrm{mK}$.

- Despite the large uncertainties, the Square Kilometer Array will have enough sensitivity to detect the signal in any one of these scenarios.

- We found that for all $\sim 200$ models the shape of the global 21-cm signal is universal. All of the signals exhibit the absorption trough. However, the late-time emission signal may not be present of X-ray heating is inefficient.

- Depending on the details of astrophysics, different sources of fluctuations could dominate at every given epoch. The dominant contribution could come from density and velocity, Ly $\alpha$ radiation, fluctuations in the heating rate, and/or ionization.

- Usually the effect of density fluctuations is assumed to be sub-dominant; however, we showed that this is not always the case and density can play a significant (and even leading) role.

- We found out that the slope of the power spectrum is much easier to analyze than the power spectrum itself. It shows universal redshift dependence and its sign can be either positive or negative. In particular, at its low-redshift minimum the slope is negative if the SED of X-ray sources is hard.

- Astrophysical properties can be extracted from either the global signal, the power spectrum or the slope of the power spectrum. We found several model-independent relations between the features of each type of the signal and the underlying astrophysical parameters. Moreover, we point out model-independent relations between the features of the global signal and the power spectrum (its slope). These relations can be used to verify future detection.

- Finally, we check in which cases the 21-cm signal can be used as a direct measure of $\tau$ (as is noted by Liu et al. (2016)). It turns out that when the peak power of ionizing fluctuations in the 21-cm signal is below $100 \mathrm{mK}^{2}$ the peak power traces the ionization fraction, $x_{i o n}$, at the redshift of the peak. On the other hand, when the peak power is large, this is a signal of weak X-ray heating which extends throughout reionization. In these cases, heating and ionization happen simultaneously and it is hard to extract the ionization fraction directly.

Motivated by the interesting results of this parameter study, we ran $\sim 20000$ more models on the Odyssey cluster at Harvard, varying the slope of the SED and the minimal energy of X-rays in addition to the 5 key parameters described above ( 7 parameters in total). This bank of models is used to create a mapping between the parameters and the global 21-cm signal ?.

\section{A Note on Fast Radio Bursts}

Fast Radio Bursts (FRBs) might provide a new way to probe the high redshift Universe. At the moment only $25 \mathrm{FRBs}$ are publicly known. They were detected by several radio telescopes (PARKES, GBT, UTMOST, ARECIBO, ASKAP) and in different frequency bands between $0.7 \mathrm{GHz}$ and $1.4 \mathrm{GHz}$. One of the FRBs was localized to a dwarf metal-poor galaxy at $z \sim 0.2$, indicating that FRBs (at least a sub-population) are of cosmological origins Tendulkar et al. (2017).

As an FRB propagates from the host galaxy to the observer through the ionized intergalactic medium, it is dispersed. The dispersion measure (DM) is defined as the integrated electron density along the line of sight. Therefore, if FRBs existed before 
EoR, their DM would saturate and would be the same for all FRBs at redshifts prior to EoR. In fact, DM can be directly related to $\tau$. Because the measurement of DM for high-redshift FRBs can be done much more precisely than that of $\tau$ from the CMB, FRBs offer a way to avoid the optical depth nuisance (poor measurement of $\tau$ degrades the precision with which other cosmological parameters can be measured Fialkov \& Loeb (2016)). If FRBs exist out to high redshifts, SKA will be able to detect them in large quantities Fialkov \& Loeb (2017).

\section{References}

Brorby, M., Kaaret, P., Prestwich, A., \& Mirabel, I. F., 2016, MNRAS, 457, 4081

Cohen, A., Fialkov, A., Barkana, R., \& Lotem, M., 2017, MNRAS, 472, 1915

Cohen, A., Fialkov, A., \& Barkana, R., 2017, submitted

Cohen, A., Fialkov, A., Barkana, R., \& Monsalve, R., in prep.

Fialkov, A., Cohen, A., Barkana, R., \& Silk, J., 2017, MNRAS, 464, 3498

Fialkov, A., \& Loeb, A., 2017, ApJ, 846, 27

Fialkov, A., \& Loeb, A., 2016, JCAP, 05, 004

Fialkov, A., Barkana, R., \& Visbal, E., 2014, Nature, 506, 197

Field, G. B. 1958, Proceedings of the IRE, 46, 240

Fragos, T., Lehmer, B. D., Naoz, S., Zezas, A., \& Basu- Zych, A., 2013, ApJ, 776, L31

Liu, A., Pritchard, J. R., Allison, R., Parsons, A. R., Seljak, U., \& Sherwin, B. D., 2016, 2016, PRD, 93, 3013

Miranda, V., Lidz, A., Heinrich, C. H., \& Hu, W., 2017, MNRAS, 467, 4050

Monsalve, R., \& Fialkov, A., et al., 2017, in prep.

O'Shea, B. W., Wise, J. H., Xu, H., \& Norman, M. L., 2015, ApJ, 807, 12

Oesch, P. A., Brammer, G., van Dokkum, P. G., Illingworth, G. D., Bouwens, R. J., et al., 2016, ApJ, 818, 129

Pacucci, F., Mesinger, A., Mineo, S., \& Ferrara, A., 2014, MNRAS, 443, 678

Planck Collaboration, Aghanim, N., Ashdown, M., et al., 2016, A\&A, 596, A107

Pober, J. C., Ali, Z. S., Parsons, A. R., et al., 2015, ApJ, 809, 62

Price, D. C., Greenhill, L. J., Fialkov, A., Bernardi, G., Garsden, H. et al., 2017, submitted

Singh, S., Subrahmanyan, R., Udaya S. N., Sathyanarayana Rao, M., \& Fialkov, A., 2017, ApJ, 845,12

Singh, S., Subrahmanyan, R., Udaya S. N., Sathyanarayana Rao, M., \& Fialkov, A., 2017, submitted

Tegmark, M., Silk, J., Rees, M., Blanchard, A., Abel, T., \& Palla, F., 1997, ApJ, 474, 1

Tendulkar, S. P., Bassa, C. G., Cordes, J. M., Bower, G. C., Law, C. J. et al., 2017, ApJ, 834L, 7

Wise, J. H., Demchenko, V. G., Halicek, M. T., Norman, M. L., Turk, M. J., Abel, T., \& Smith, B. D., 2014, MNRAS, 442, 2560

Wouthuysen, S. A. 1952, AJ, 57, 31

Xu, H., Wise, J. H., Norman, M. L., Ahn, K., \& O'Shea, B. W., 2016, ApJ, 833, 84 